\title{
On Quantitative Analysis of Inclusions forming Cloud Group using EPMA
}

\author{
By Yoshimoto Wanibe* and Kokichi Sano*
}

\begin{abstract}
Electron probe X-ray microanalysis was performed to confirm whether the inclusions comprising the stratiform inclusions group are alumina as shown previously by microscopic observations. The results were compared with those of analysis of the standard specimen in which pure alumina powder was embedded in pure iron by pressing and sintering. The results may be summarized as follows;

(1) The inclusions forming the stratiform group were alumina at large.

(2) The inclusions of less than about $5.5 \mu$ in diameter are effected by the matrix at the electron beam diameter of about $1.5 \mu$.

(3) Alumina inclusions could be successfully determined by reckoning the alumina content in the inclusions backward from the relative intensity of iron.

(4) The aluminum contents in the matrix which were corrected by using Philibert's equation have good agraement with those estimated from the relative intensity of iron. But Birks' correction deviates from the actual data.

(5) The alumina content in the inclusions which was corrected by using Birks' equation differs greatly from that calculated directly from the relative intensity of aluminum, but agrees relatively well with that estimated indirectly from the wüstite content.
\end{abstract}

(Received May 10, 1968)

\section{Introduction}

We have already investigated the effect of the initial oxygen content in liquid iron and the reaction time on the behavior of the stratiform inclusions group formed when aluminium is dropped onto the static liquid iron, and have discussed the nucleation and growth mechanism of the inclusions which make up the group (1) (3). In those reports, we used the electron probe X-ray microanalyzer to determine the aluminum content in the matrix of the specimens. However, the X-ray radiated from the elements with low atomic numbers, such as aluminum, is generally absorbed by the surrounding iron atoms, etc., so that the analytical results show much lower values than the actual weight fractions. More-

* Department of Metallurgy, Faculty of Engineering, Nagoya-University, Nagoya, Japan.

(1) Y. Wanibe and K. Sano : J. Japan Inst. Metals, 30 (1966), 674; Trans. JIM, 8 (1967), 75.

(2) Y. Wanibe and K. Sano : J. Japan Inst. Metals, 31 (1967), 199; Trans. JIM, 9 (1968), awaiting publication.

(3) Y. Wanibe and K. Sano : J. Japan Inst. Metals, 31 (1967), 795; Trans. JIM, pэnding publication. over, owing to the lack of information necessary for the correction of our experimental data, we estimated the aluminum content in the matrix by reckoning backward from the relative intensity of iron, because, even if our specimens contained oxygen atoms, iron atoms and aluminum atoms, the oxygen content was negligibly small as compared with the other two elements. Also, as the formed inclusions comprising the stratiform inclusions group were very few and a local analysis seemed to be necessary, the usual chemical analysis could not be applied. So it was decided by microscopic observation that the inclusions were alumina ${ }^{(4)}$.

Then, the composition of the inclusions forming the stratiform inclusions group was investigated to confirm whether the formed inclusions are alumina as predicted in the previous reports. The analytical results of the composition of inclusions, especially low atomic number elements, by the microanalyzer were not reliable. However, the microanalysis was excellent in the local analysis. In order to clarify the results, the actually analyzed data of the inclusions comprising the stratiform inclusions group were also

(4) F. B. Pickering : J. Iron and Steel, 30 (1957), 3. 
compared with the results obtained from standard specimens in which pure alumina powder was embedded in pure iron by pressing or by pressing and sintering.

\section{Experimental Apparatus and Procedure}

The experimental apparatus and procedure were the same as those described in the previous report(1). However, in order to discuss in detail the results obtained by microanalysis, standard specimens were produced by burying pure alumina powder into pure iron by means of pressing or pressing and sintering. The pure alumina powder used was Merk's reagent standardized for chromatographic adsorption analysis. At first, pure iron used as the matrix of the standard specimens was melted. About $300 \mathrm{~g}$ of electrolytic iron was put into a $50 \phi \times 38 \phi \times 60 \mathrm{~mm}$ flat-bottomed porous magnesia crucible. Melting was carried out under a mixed atmosphere of refined argon gas and hydrogen gas maintained at a ratio of about $1: 1$, by using a high frequency wave induction furnace of the $15 \mathrm{~kW} 430 \mathrm{kC}$ vacuum tube type. The melt was reduced for about two hours at approximately $1600^{\circ} \mathrm{C}$ until the oxygen content in liquid iron reached $0.001 \%$ $\sim 0.002 \%$. Then the hydrogen gas was cocked off, and then the hydrogen dissolved in the liquid iron was removed by diffusion. Then the furnace current was cut off and the melt was solidified and cooled.

Two specimens of $15 \times 10 \times 6 \mathrm{~mm}$ were cut from the pure iron block, and the surfaces of the specimens were polished by the ordinary mechanical method including buff-polishing. Then, a suitable amount of alumina powder was pressed into the pure iron specimens to make standard specimens.

One of these standard specimens was put into a $26 \phi \times 22 \phi \times 60 \mathrm{~mm}$ flat-bottomed semi-fused alumina crucible and then heated for about half an hour at approximately $1100^{\circ} \mathrm{C}$ using a high frequency wave induction furnace of the $20 \mathrm{~kW}$ mercury gap type, under the mixed atmosphere of refined argon gas and refined hydrogen gas maintained at a ratio of about 10:1. Then, the furnace current was cut off and the specimen was cooled. After the surface of the specimen was polished to some extent with diamond paste, it was used as a standard specimen for microanalysis.

The nonmetallic inclusions in the specimens and the standard specimens were analyzed quantitatively by the microanalyzer, using both line scanning analysis at a speed of $10 \mu / \mathrm{min}$ and point analysis.

\section{Experimental Results}

First, the stratiform inclusions group in the specimen used for microanalysis was observed by a microscope. The result is shown in Photo. 1, where the aluminum-rich part is on the upper side and the iron-rich part on the lower side. The vertical line in the center is the contamination formed during the quantitative analysis of the matrix with the microanalyzer. This specimen was obtained by dropping

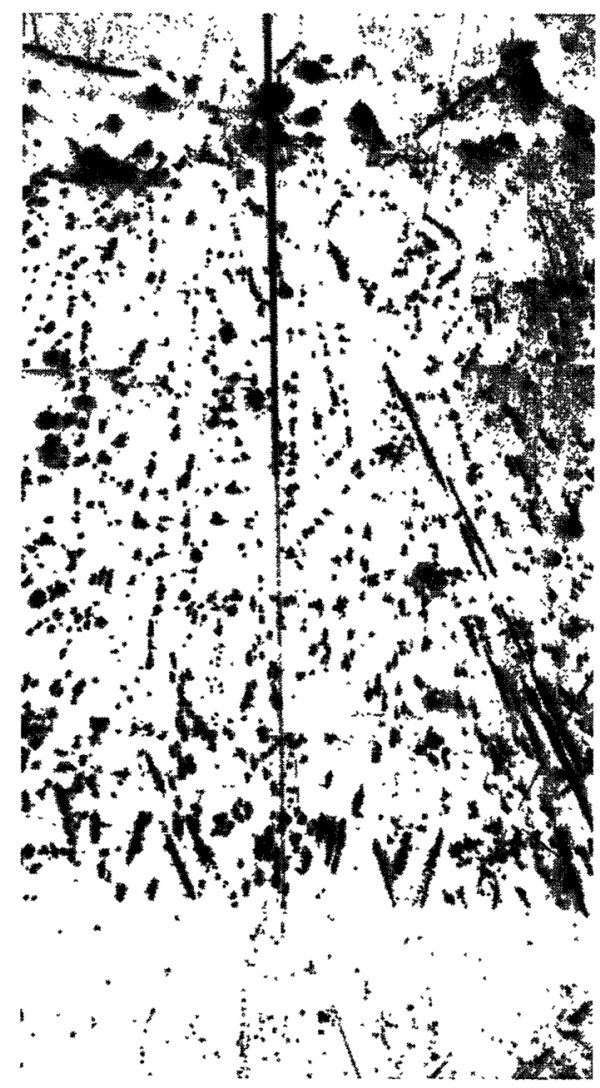

Photo. 1 Microphotograph of the inclusions group in the specimen, containing initial oxygen content of $0.0848 \%$ and reacted for 400 sec (non-etched, $\times 42)$

aluminum onto the static liquid iron containing $0.0848 \%$ oxygen and then deoxidizing the liquid iron with aluminum for 400 sec. A stratiform inclusions group was formed in the region containing $2.8 \%$ $6.8 \%$ aluminum in the matrix, with a width of about $1.8 \mathrm{~mm}$ at a distance of about $6.2 \mathrm{~mm}$ to $8.0 \mathrm{~mm}$ from the Grube's contact surface. These measurements were estimated from the mean value of the thickness of the group measured by the microscope, and the analysis of the matrix by the microanalyzer ${ }^{(1)}$.

Some inclusions were chosen at random from the stratiform inclusions group formed in the specimen, Photo. 1, and then exposed to the electron beam to analyze their compositions by using techniques of line scanning analysis at a speed of $10 \mu / \mathrm{min}$ and point analysis. The results are shown in Table 1. At a glance, there are some noteworthy points. First, the total weight fraction of iron and aluminum shown in the third row of the weight fraction of the matrix, deviated slightly to lower values, i.e. from $92 \%$ to $98 \%$. But, there is a regularity in this deviation. Its total has a tendency to decrease with increase in weight fraction of aluminum. In order to investigate the composition of the inclusions, the iron and aluminum weight fractions obtained from the relative intensities of $\mathrm{X}$-rays radiated from the inclusions were recalculated as wüstite and alumina fractions, respectively. The results are shown in the second and fourth rows of the weight fraction of the inclusions in Table 1 . The total weight fractions of wüstite and alumina shown in the fifth row were 
Table 1 EPMA analysis of inclusions

\begin{tabular}{|c|c|c|c|c|c|c|c|c|c|c|c|}
\hline \multirow{2}{*}{$\begin{array}{c}\text { Size } \\
\mu\end{array}$} & \multicolumn{5}{|c|}{ Weight fraction in matrix (\%) } & \multicolumn{6}{|c|}{ Weight fraction in inclusions (\%) } \\
\hline & $\mathrm{Fe}$ & $\mathrm{Al}$ & total & $\underset{\mathrm{cal}}{\mathrm{Al}}$ & $\begin{array}{l}\text { total } \\
\text { cal }\end{array}$ & $\mathrm{Fe}$ & $\mathrm{FeO}$ & $\mathrm{Al}$ & $\mathrm{Al}_{2} \mathrm{O}_{3}$ & total & $\begin{array}{l}\mathrm{Al}_{2} \mathrm{O}_{3} \\
\text { est. }\end{array}$ \\
\hline 12 & 97.4 & 0.505 & 97.9 & 2.12 & 99.5 & 6.50 & 8.40 & 18.9 & 35.3 & 43.7 & 91.6 \\
\hline 7 & 96.9 & 0.547 & 97.4 & 2.30 & 99.2 & 6.65 & 8.65 & 16.2 & 30.8 & 39.5 & 91.4 \\
\hline 18 & 97.2 & 0.703 & 97.9 & 2.90 & 100 & 1.28 & 1.65 & 26.0 & 49.1 & 50.8 & 98.4 \\
\hline 5 & 96.5 & 0.797 & 97.3 & 3.35 & 99.9 & 30.8 & 39.8 & 16.7 & 30.0 & 69.8 & - \\
\hline 11 & 95.7 & 0.916 & 96.6 & 3.97 & 99.7 & 2.58 & 3.33 & 18.0 & 34.1 & 37.4 & 96.7 \\
\hline 8.5 & 93.7 & 1.23 & 94.9 & 5,47 & 99.2 & 6.80 & 8.85 & 21.8 & 40.8 & 49.7 & 91.2 \\
\hline 9 & 93.7 & 1.44 & 95.1 & 6.05 & 99.8 & 34.7 & 44.8 & 9.04 & 17.1 & 61.9 & 55.2 \\
\hline 10 & 94.1 & 1.66 & 95.8 & 7.43 & 102 & 1.20 & 1.55 & 21.1 & 39.9 & 41.5 & 98.5 \\
\hline 20 & 90.7 & 1.85 & 92.6 & 7.89 & 98.6 & 7.00 & 9.05 & 16.9 & 31.8 & 40.9 & 91.0 \\
\hline
\end{tabular}

scattered remarkably from about $39 \%$ to $70 \%$. Moreover, there is little regularity in the scattering. It seems, however, that the total weight fraction of wïstite and alumina becomes larger with increasing relative intensity of radiation from iron. Because of the ambiguity of this, it cannot be decisively determined whether the inclusions are alumina or spinel hercynite.

Next, the standard specimens, pure alumina powder embedded in pure iron, were used for microanalysis and the relative intensities of radiation from alumina were examined by using the same method as in the determination of the compositions of the inclusions. Of the two standard specimens, the one in which alumina powder was embedded in pure iron by pressing could not be buff-polished because of the removal of alumina particles from the specimen. Since buff-polishing was not done before electron probe $\mathrm{X}$ ray analysis, the surface of the inclusions was exceedingly uneven so that the $\mathrm{X}$-rays from the inclusions were scattered in all directions resulting in the lower scattering weight fractions of iron and aluminum. The other standard specimen, obtained by pressing and sintering, could be buff-polished and fitted for microanalysis. Thus, this specimen was used as the standard specimen for the quantitative analysis of the inclusions by the microanalyzer. The electron beam emitted ranged from $1.2 \mu$ to $1.7 \mu$ in diameter for electron probe X-ray analysis. But, as the atoms around the target of the electron beam is naturally excited, it is considered that the analytical results of the small inclusions contain the effect of the matrix. Therefore, in order to establish a condition in which the matrix has no effect on the analytical results of the inclusions, the analytical results of alumina in the standard specimen are diagrammatically shown in Fig. 1 as a function of the diameter. Here the diameters of the inclusions were estimated from the charts obtained by line scanning analysis of the specimen at a speed of $10 \mu / \mathrm{min}$, because, even though this method cannot give us the depths of the inclusions, there are no other adequate methods. In Fig. 1, the open circles show the alumina content calculated from the relative intensity of aluminum, and the solid circles indicate the alumina content by reckoning backward from the relative intensity of iron; i.e. the rest of the wüstite content estimated from

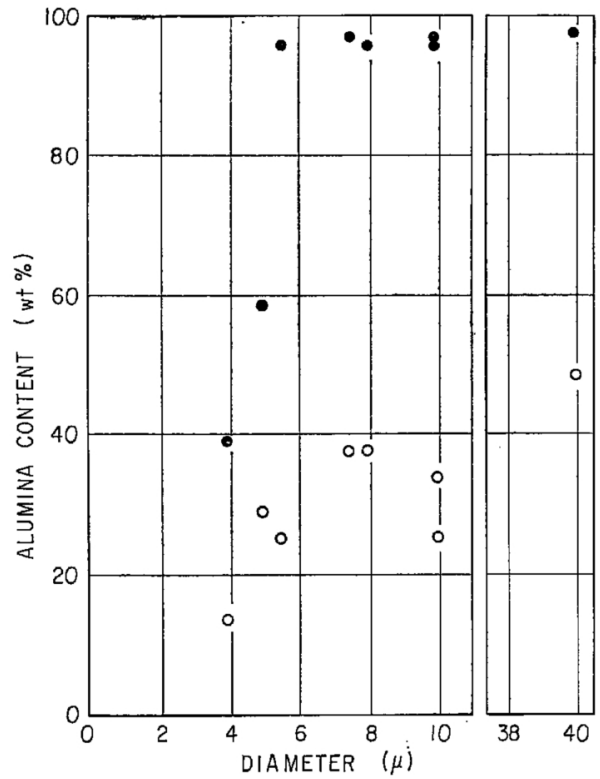

Fig. 1 Effect of the diameter of inclusions on EPMA analysis (result of the standard specimen)

the relative intensity of iron was treated as the alumina content. The horizontal line in Fig. 1 shows the alumina content in spinel hercynite. According to this figure, the alumina content estimated from the relative intensity of aluminum was extensively scattered in the region between $13 \%$ and $50 \%$ which was lower than expected. On the other hand, the alumina content in the inclusions, reckoned backward from the relative intensity of iron, becomes a fixed value and ranged from about $96 \%$ to $98.5 \%$ against the diameters of more than $5.5 \mu$. Thus, in order to reasonably determine whether the inclusions are alumina or spinel hercynite, the inclusions with diameters of more than $5.5 \mu$ must be analyzed, and the alumina content in the inclusions must be calculated backward from the relative intensity of iron.

Next, the alumina content in the inclusions was also reckoned backward from the relative intensity of iron and the results are shown in the sixth row of the weight fraction of the inclusions in Table 1. This table shows that the inclusions comprising the stratiform inclusions group are alumina at large, thus confirming the microscopic results obtained previously. It is possible, however, that the inclusions 
shown in the seventh line may be spinel hercynite.

The inclusions comprising the stratiform inclusions group were also exposed to the electron beam and the relative intensity of the $\mathrm{X}$-rays from the iron atoms was read on the meter. The results showed that most of the inclusions forming the group were alumina. Especially, the inclusions of low aluminum contents in the group, namely those in the marginal limit of diffusion of aluminum, seems to be alumina. And it is within a probability of less than 5\% that the inclusions in the other parts of the group are spinel hercynite.

Moreover, an attempt was made to identify the composition of the isolated inclusions ahead of the end of the stratiform inclusions group, i.e. the inclusions in the region of much lower aluminum contents in the matrix. But the inclusions were so small that their compositions could not be investigated.

\section{Discussion}

First, we would like to discuss the results obtained by microanalysis of the aluminum content in the matrix.

The matrix in the specimens, including those used in the previous reports, was analyzed. Two aluminum contents were estimated from the relative intensity of aluminum and by reckoning backward from the intensity of iron, as stated above. The relation between these contents is shown in Fig. 2. The relation was approximated as a straight line,

$$
C_{\text {est }}=4.20 C_{\text {int }},
$$

where $C_{\text {est }}=$ the aluminum content estimated from the relative intensity of iron,

$C_{\text {int }}=$ the aluminum content calculated from the relative intensity of aluminum.

The line (1) in Fig. 2 represents eq. (1).

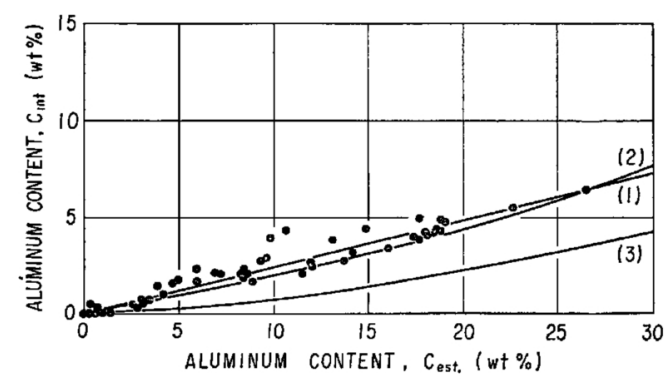

Fig. 2 Discussion on the correction of EPMA analysis

Then, the absorption correction was calculated by Philibert's equation for the approximate absorption correction of the multi-components system(5). The result obtained is shown by the line (2) in Fig. 2, which indicates that there is good agreement between the aluminum content estimated from the relative intensity of iron and that calculated by using Philibert's equation.

(5) J. Philibert : The American Society for Testing and Materials, (1962); Process Third International Symposium of X-Ray Optics and X-Ray Microanalysis, (1963).
Moreover, Birks' equation used for the absorption correction of the multi-components system(6), was $^{(6)}$ applied for this experimental system. But, as the absorption function used for this equation is still unknown in the whole region of the aluminum-ironoxygen system, the function was extrapolated, assuming that according to the form of the function, it decreases logarithmically in the region of large parameter, $\mu^{\prime} \operatorname{cosec} \psi$, as shown in Fig. 3. Birks' absorp-

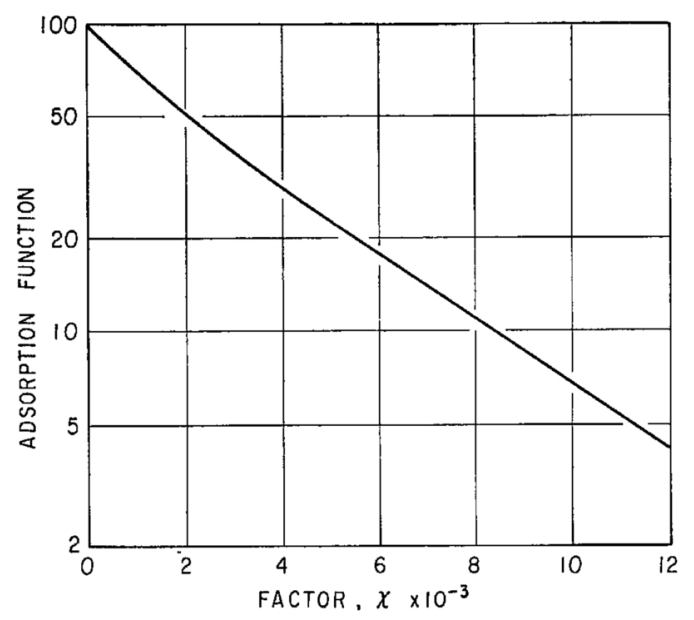

Fig. 3 Relation between absorption function and factor $\chi$ $\left(=\mu^{\prime} \operatorname{cosec} \psi\right)$

tion correction estimated in this way is shown by line (3) in Fig. 2. According to Fig. 2, the aluminum content corrected by Birk's equation has the same tendency as that estimated from the relative intensity of iron, but it has a little deviation. What causes this disagreement is still unknown, and this problem has to be further investigated. Since the total of the aluminum and iron contents in the matrix was in excess of $100 \%$ when Birks' correction was applied for this system in the present work, the aluminum content in the matrix was corrected by using Philibert's equation. As shown in Table 1, the corrected aluminum content is shown in the fourth row of the weight fraction of matrix, and the corrected total weight fraction of the iron and aluminum contents in the fifth row.

Next, we would like to discuss the results obtained by quantitative microanalysis of the inclusions. First, according to the equilibrium phase diagram of the wüstite-alumina system shown in Fig. $4^{(7)}$, the solubility of wüstite in alumina is about $2 \%$, forming a solid solution at our experimental temperature $1550^{\circ} \mathrm{C}$. Spinel hercynite also seems to contain about $41 \%$ wuistite. Then, in accordance with the diagram, the alumina contents in the inclusions with diameters of more than $5.5 \mu$ in Table 1 were rearranged from the viewpoint of the aluminum content in the matrix and the distance from the Grube's contact surface. The results are shown in Fig. 5. Here the open circles show the alumina contents in the inclusions estimated from the relative intensity of aluminum,

(6) J. S. Bards : Electron Probe Microanalysis, John Wiley Sons, (1963), N.Y., London.

(7) W. A. Fischer and A. Hoffmann : Arch. Eisenhütt., 27 (1956), 343. 


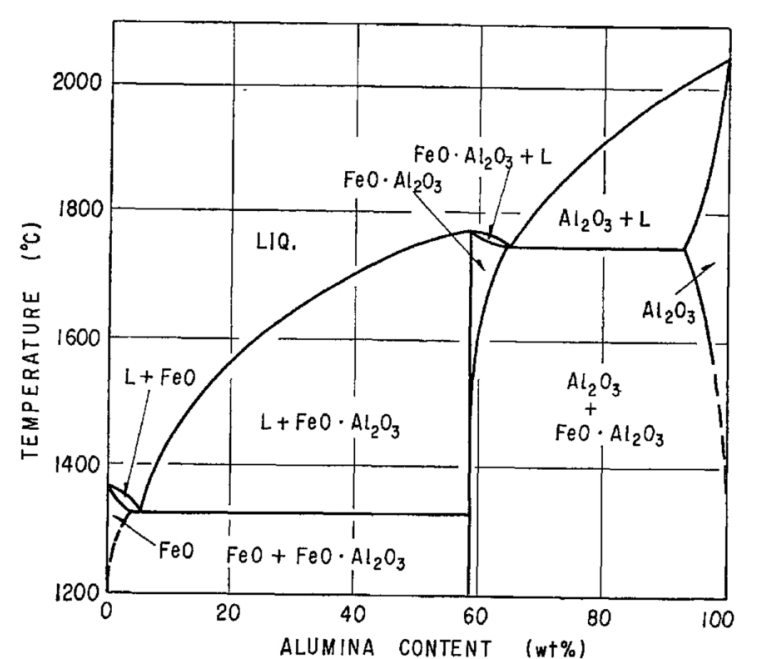

Fig 4 Diagram of the $\mathrm{FeO}-\mathrm{Al}_{2} \mathrm{O}_{3}$ systэm

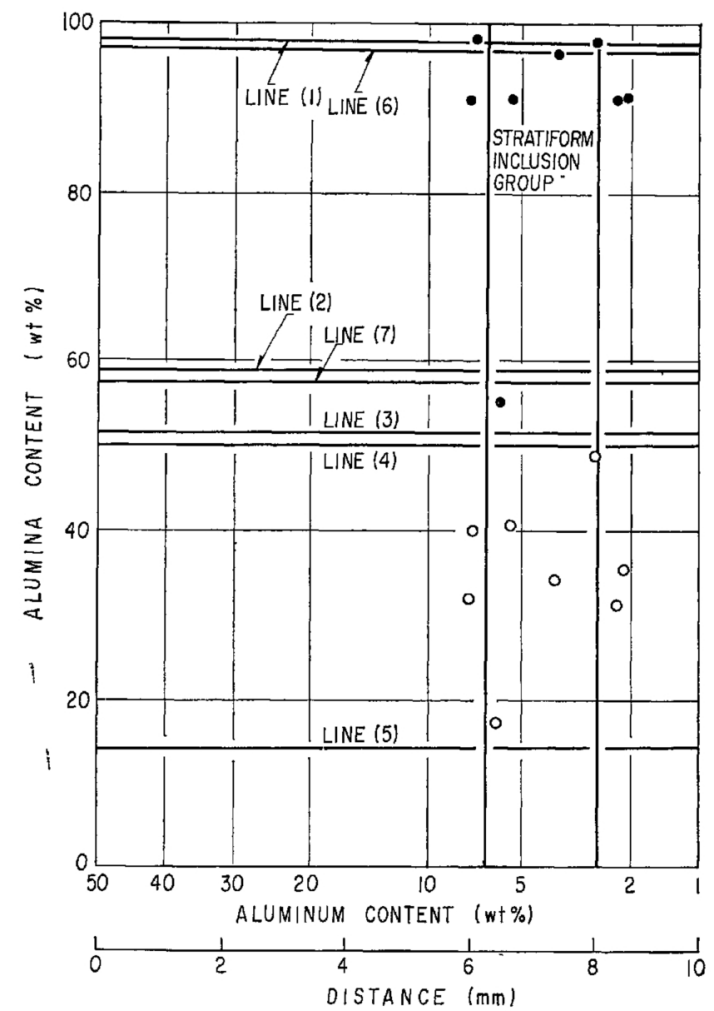

Fig. 5 Composition of inclusions depending on aluminum content in matrix and position

and the solid circles show the alumina content reckoned backward from the relative intensity of iron. The region of alumina and the line of spinel hercynite obtained from Fig. 4 are drawn in Fig. 5; the alumina region extends over alumina contents higher than line (1), and the spinel hercynite line is given by line (2). However, as elements of low atomic number, such as aluminum, need the absorption correction as stated above, the corrected alumina content in alumina and spinel hercynite were calculated by using Birks' equation for the absorption correction of the multi-components system and the extrapolated values shown in Fig. 3. The region of alumina and the alumina content in spinel hercynite were calculated from the relative intensity of $\mathrm{X}$-rays from aluminum to be between lines (3) and (4), and to be on line (5) in Fig. 5, respectively. On the other hand, the region of alumina and the alumina content in spinel hercynite were also reckoned on the basis of the relative intensity of $\mathrm{X}$-rays from iron to extend beyond line (6) and on line (7), respectively. According to Fig. 5, the corrected alumina content in alumina has good agreement with that reckoned backward indirectly by using the wüstite content, probably because of the low solubility of wüstite in alumina. Thus, the kind of inclusions could be determined relatively well by the microanalyzer, even if some error occurred during the measurement, but it is difficult to determine the composition of the inclusions. Moreover, the relative intensity of aluminum was so extensively scattered that the alumina content in the inclusions directly calculated from it could not be compared in detail with the correction of the Birks' equation. What causes this scattering is still unknown and should be further investigated.

\section{v. Summary}

An aluminum bar was dropped onto liquid iron containing oxygen, with a negligibly small thermal convection, and the inclusion comprising the stratiform inclusions group formed during the deoxidation of liquid iron with aluminum were analyzed by the microanalyzer to confirm the results in the previous reports. During the microanalysis, the results were compared with the analysis of the standard specimen in which pure alumina powder was embedded in pure iron by pressing and sintering. The results can be summarized as follows:

(1) According to the microanalytical results of the alumina standard specimen, the inclusions less than about $5.5 \mu$ in diameter are affected by the matrix when the electron beam diameter is about $1.5 \mu$. It also can be determined whether the inclusions are alumina or not, by reckoning the alumina content in the inclusion backward from the relative intensity of iron, but it is difficult to do so by the ealculation from the intensity of the aluminum because of the pronounced scattering.

(2) The inclusions comprising the stratiform inclusions group were analyzed by the microanalyzer to be alumina inclusions at large. Especially, at a lower aluminum content in the matrix, all the formed inclusions were alumina. This confirms the result obtained in the previous reports.

(3) Philibert's and Birks' equation for the absorption correction was applied to the microanalytical results of the matrix, respectively. The Philibert's correction has good agreement with the aluminum contents in the matrix which were reckoned backward from the relative intensity of iron, but the Birks' correction has a little deviation.

(4) Birks' equation was applied in the case of microanalysis of the alumina inclusions. The corrected alumina content agrees relatively well with that reckoned backward indirectly from the wüstite content, probably because of the low solubility of wüstite in alumina. The scattering of alumina contents, calculated from the intensity of aluminum, however, made comparison impossible. What causes this scattering is still unknown. 